\title{
Emission of Self-Assembled CdTe/ZnTe Quantum Dot Samples with Different Cap Thickness
}

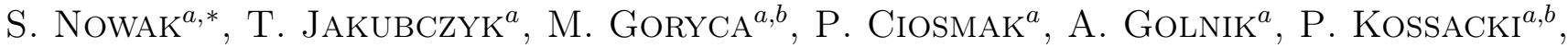 \\ P. WOJNAR ${ }^{c}$ AND J.A. GAJ ${ }^{a}$ \\ ${ }^{a}$ Institute of Experimental Physics, University of Warsaw, Hoża 69, 00-681 Warsaw, Poland \\ ${ }^{b}$ Grenoble High Magnetic Field Laboratory, CNRS, Grenoble, France \\ ${ }^{c}$ Institute of Physics, Polish Academy of Sciences, al. Lotników 32/46, 02-668 Warsaw, Poland
}

\begin{abstract}
Photoluminescence measurements on $\mathrm{CdTe} / \mathrm{ZeTe}$ self-assembled quantum dot samples with different cap thickness values $(18-110 \mathrm{~nm})$ were performed at $1.8 \mathrm{~K}$ at varying excitation levels. The shape of macrophotoluminescence spectra did not altered notably with the excitation power. The spectra exhibited interference fringes related to the total barrier thickness. Simulation of the fringes confirmed the barrier thickness determined during the growth. The minimal amplitude of the fringes was observed for the cap thickness corresponding approximately to a quarter of the emission wavelength in the barrier material. Maximum emission intensity occurred for the largest thickness of the cap, i.e., $110 \mathrm{~nm}$. We attribute this result to the influence of the surface recombination centers.
\end{abstract}

PACS numbers: 78.55.Et, 78.67.Hc

\section{Introduction}

The idea of tuning the structure of a sample to optimize the light-matter coupling may be important for many applications of semiconductors in light emission or detection devices. A strong influence of the outer barrier (cap) thickness on the form of reflectivity spectra has been reported, e.g., for single $\mathrm{GaAs} /(\mathrm{Ga}, \mathrm{Al}) \mathrm{As}$ quantum wells [1]. The tuning of the cap thickness may be also tested as a tool to optimize the light emission. A natural expectation would be that the emission of a quantum well or a quantum dot could be enhanced by selecting a quarter-wavelength outer barrier (cap) thickness. This value corresponds to a destructive interference for emission from the quantum structure towards the substrate, and thus may lead to an enhancement of the probability of light extraction from the sample.

This work was planned to test the practical utility of such an idea, applied to self-assembled CdTe/ZnTe quantum dot (QD) samples.

\section{Samples and experiment}

A series of samples was grown by molecular beam epitaxy on GaAs substrates. After the deposition of a $3.8 \mu \mathrm{m}$ thick ZnTe buffer, which served as the inner barrier, a self-assembled CdTe QD layer was grown, followed

\footnotetext{
* Present address: Department of Physics, University of Fribourg, CH-1700 Fribourg, Switzerland.
}

by a cap (outer barrier). The cap thickness was varied in the range from 18 to $110 \mathrm{~nm}$.

Photoluminescence (PL) measurements have been performed at $T=1.8 \mathrm{~K}$ on all the samples at varying excitation levels. The excitation wavelength was $488 \mathrm{~nm}$.

\section{Experimental results}

The obtained spectra of the QD ensemble photoluminescence extended in the range between 550 and $650 \mathrm{~nm}$ (see Fig. 1). They exhibited interference fringes related to the total barrier thickness. The shape of the ensemble spectra weakly depended on the excitation power. All samples demonstrated also a weak dependence of the

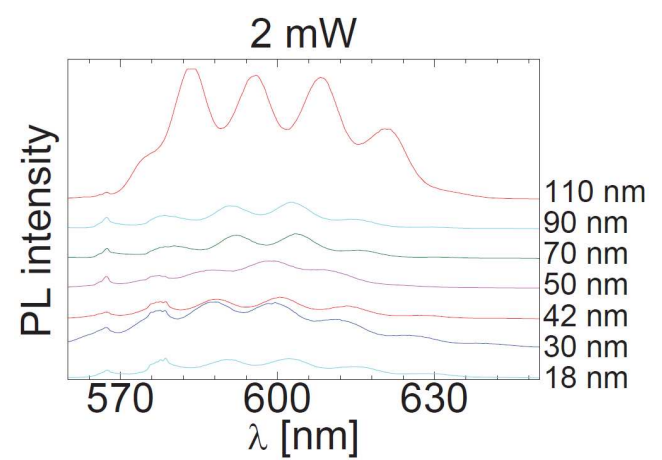

Fig. 1. Macrophotoluminescence spectra of the samples measured at excitation power of $2 \mathrm{~mW}$. Cap thickness values indicated for each curve. 


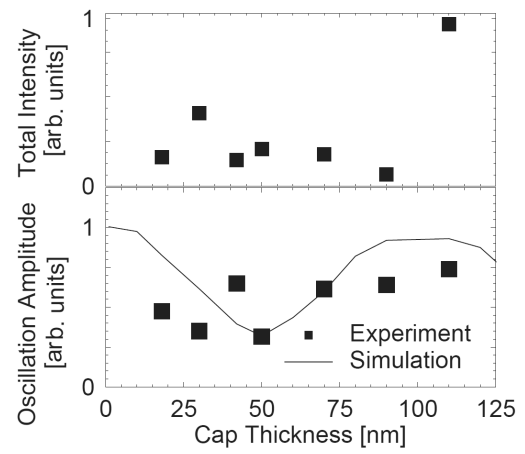

Fig. 2. Integrated intensity of measured PL as a function of the cap thickness (top) and experimental amplitude of the interference fringes compared to simulation results (bottom).

PL spectrum on the excitation spot position, which evidenced a good homogeneity of the samples.

The amplitude of the fringes was minimal for the cap thickness of $50 \mathrm{~nm}$, corresponding approximately to a quarter of the emission wavelength in the barrier material (see Fig. 2, bottom plot).However, this cap thickness did not correspond to a maximum emission intensity, which occurred for the largest thickness of the cap, i.e., $110 \mathrm{~nm}$ (see Fig. 2, top).

In microphotoluminescence measurements (not shown), typical emission lines [2] of neutral excitons, charged excitons, and biexcitons were identified. Their relative intensities exhibited a typical excitation power dependence.

\section{Photoluminescence simulations}

Simulations of the PL spectra were performed for each cap thickness. Isotropic light emission of the QD ensemble with a Gaussian spectrum was assumed. The simulation took into account interference in the ZnTe layer.

TABLE

The output parameters of the PL simulation.

\begin{tabular}{c|c|c|c|c}
\hline \hline \multicolumn{2}{|c|}{ Sample structure } & \multicolumn{3}{|c}{ QD emission } \\
\hline $\begin{array}{c}\text { cap } \\
{[\mathrm{nm}]}\end{array}$ & $\begin{array}{c}\text { buffer } \\
{[\mathrm{nm}]}\end{array}$ & $\begin{array}{c}\text { position } \\
{[\mathrm{nm}]}\end{array}$ & $\begin{array}{c}\text { width } \\
{[\mathrm{nm}]}\end{array}$ & $\begin{array}{c}\text { intensity } \\
\text { [arb.u.] }\end{array}$ \\
\hline 18 & 3412 & 597 & 48 & 17 \\
30 & 3390 & 593 & 48 & 40 \\
42 & 3400 & 595 & 43 & 16 \\
50 & 3682 & 597 & 37 & 22 \\
70 & 3343 & 597 & 37 & 18 \\
90 & 3330 & 597 & 36 & 19 \\
110 & 3460 & 598 & 42 & 100
\end{tabular}

The input data for the simulation were the cap thickness and the refractive indexes of ZnTe [3] and GaAs [4].

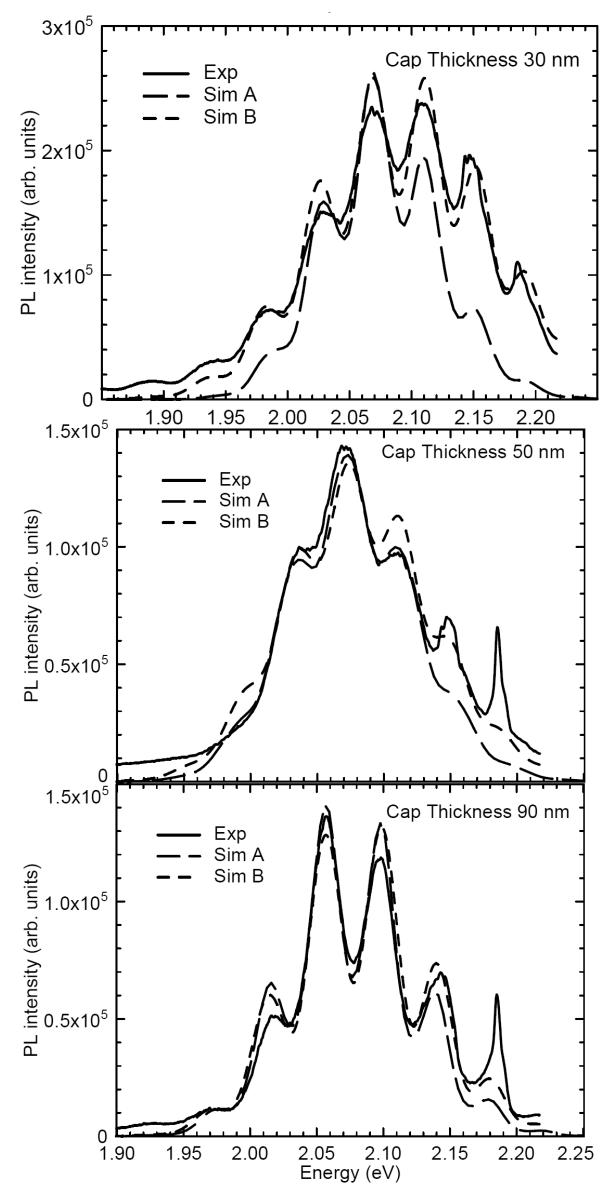

Fig. 3. Experimental PL spectra compared with two types of simulations for different values of the cap thickness.

Free parameters, adjusted to fit experimental spectra, were the parameters of the Gaussian function describing the QD emission and the ZnTe buffer thickness. The simulations were performed in two versions: with common values of the position and width of the Gaussian curve describing the QD emission (simulation A) and with these parameters adjusted individually for each sample (simulation B).

Examples of the simulations are shown in Fig. 3. As visible in the figure, common Gaussian parameters (simulation A) are not sufficient to achieve a correct description of the experimental spectra. The parameters of simulation B are collected in Table.

\section{Discussion}

As expected the amplitude of the fringes is minimal for the cap thickness of $50 \mathrm{~nm}$ (see Fig. 2). This corresponds to a destructive interference between direct downward emission of the QD plane and its upward emission reflected from the surface. However, this cap thickness does not correspond to the maximum PL intensity which is observed for the $110 \mathrm{~nm}$ cap. We attribute this result 
to surface-related non-radiative recombination centers responsible for suppression of the QD's emission intensity. Their influence is expected to decrease with the cap thickness.

The interferences have little effect on total PL intensity emitted from the sample.

\section{Acknowledgments}

This work was supported by Polish Ministry of Science and Higher Education (contract financed in 2007-2010) and Marie Curie Actions (contract number MTKD-CT-2005-029671).

\section{References}

[1] X.L. Zheng, D. Heiman, B. Lax, F.A. Chambers, Appl. Phys. Lett. 52, 287 (1988).

[2] T. Kazimierczuk, J. Suffczyński, A. Golnik, J.A. Gaj, P. Kossacki, P. Wojnar, Phys. Rev. B 79, 153301 (2009).

[3] D.T.F. Marple, J. Appl. Phys. 35, 539 (1964).

[4] S. Zollner, J. Appl. Phys. 90, 515 (2001). 